\title{
Refined Bounds on the Number of Connected Components of Sign Conditions on a Variety
}

\author{
Sal Barone • Saugata Basu
}

Received: 11 April 2011 / Revised: 3 October 2011 / Accepted: 6 November 2011 /

Published online: 30 November 2011

(C) Springer Science+Business Media, LLC 2011

\begin{abstract}
Let $\mathrm{R}$ be a real closed field, $\mathcal{P}, \mathcal{Q} \subset \mathrm{R}\left[X_{1}, \ldots, X_{k}\right]$ finite subsets of polynomials, with the degrees of the polynomials in $\mathcal{P}$ (resp., $\mathcal{Q}$ ) bounded by $d$ (resp., $d_{0}$ ). Let $V \subset \mathrm{R}^{k}$ be the real algebraic variety defined by the polynomials in $\mathcal{Q}$ and suppose that the real dimension of $V$ is bounded by $k^{\prime}$. We prove that the number of semi-algebraically connected components of the realizations of all realizable sign conditions of the family $\mathcal{P}$ on $V$ is bounded by
\end{abstract}

$$
\sum_{j=0}^{k^{\prime}} 4^{j}\left(\begin{array}{c}
s+1 \\
j
\end{array}\right) F_{d, d_{0}, k, k^{\prime}}(j)
$$

where $s=\operatorname{card} \mathcal{P}$, and

$$
F_{d, d_{0}, k, k^{\prime}}(j)=\left(\begin{array}{c}
k+1 \\
k-k^{\prime}+j+1
\end{array}\right)\left(2 d_{0}\right)^{k-k^{\prime}} d^{j} \max \left\{2 d_{0}, d\right\}^{k^{\prime}-j}+2(k-j+1)
$$

In case $2 d_{0} \leq d$, the above bound can be written simply as

$$
\sum_{j=0}^{k^{\prime}}\left(\begin{array}{c}
s+1 \\
j
\end{array}\right) d^{k^{\prime}} d_{0}^{k-k^{\prime}} O(1)^{k}=(s d)^{k^{\prime}} d_{0}^{k-k^{\prime}} O(1)^{k}
$$

\section{S. Barone $\cdot$ S. Basu $(\bowtie)$}

Department of Mathematics, Purdue University, West Lafayette, IN 47906, USA

e-mail: sbasu@math.purdue.edu

S. Barone

e-mail: sbarone@math.purdue.edu 
(in this form the bound was suggested by Matousek 2011). Our result improves in certain cases (when $d_{0} \ll d$ ) the best known bound of

$$
\sum_{1 \leq j \leq k^{\prime}}\left(\begin{array}{l}
s \\
j
\end{array}\right) 4^{j} d(2 d-1)^{k-1}
$$

on the same number proved in Basu et al. (Proc. Am. Math. Soc. 133(4):965-974, 2005) in the case $d=d_{0}$.

The distinction between the bound $d_{0}$ on the degrees of the polynomials defining the variety $V$ and the bound $d$ on the degrees of the polynomials in $\mathcal{P}$ that appears in the new bound is motivated by several applications in discrete geometry (Guth and Katz in arXiv:1011.4105v1 [math.CO], 2011; Kaplan et al. in arXiv:1107.1077v1 [math.CO], 2011; Solymosi and Tao in arXiv:1103.2926v2 [math.CO], 2011; Zahl in arXiv:1104.4987v3 [math.CO], 2011).

Keywords Semi-algebraic sets $\cdot$ Sign conditions $\cdot$ Connected components $\cdot$ Betti numbers

\section{Introduction}

Let $\mathrm{R}$ be a real closed field. We denote by $\mathrm{C}$ the algebraic closure of $\mathrm{R}$. Let $\mathcal{P}$ be a finite subset of $\mathrm{R}\left[X_{1}, \ldots, X_{k}\right]$. A sign condition on $\mathcal{P}$ is an element of $\{0,1,-1\}^{\mathcal{P}}$.

The realization of the sign condition $\sigma$ in a semi-algebraic set $V \subset \mathrm{R}^{k}$ is the semialgebraic set

$$
\mathcal{R}(\sigma, V)=\left\{x \in V \mid \bigwedge_{P \in \mathcal{P}} \operatorname{sign}(P(x))=\sigma(P)\right\} .
$$

More generally, given any first order formula $\Phi\left(X_{1}, \ldots, X_{k}\right)$, the realization of $\Phi$ in a semi-algebraic set $V \subset \mathrm{R}^{k}$ is the semi-algebraic set

$$
\mathcal{R}(\Phi, V)=\{x \in V \mid \Phi(x)\} .
$$

We denote the set of zeros of $\mathcal{P}$ in $\mathrm{R}^{k}$ (resp., in $\mathrm{C}^{k}$ ) by

$$
\begin{aligned}
\operatorname{Zer}\left(\mathcal{P}, \mathrm{R}^{k}\right) & =\left\{x \in \mathrm{R}^{k} \mid \bigwedge_{P \in \mathcal{P}} P(x)=0\right\} \\
\left(\text { resp., } \operatorname{Zer}\left(\mathcal{P}, \mathrm{C}^{k}\right)\right. & \left.=\left\{x \in \mathrm{C}^{k} \mid \bigwedge_{P \in \mathcal{P}} P(x)=0\right\}\right) .
\end{aligned}
$$

The main problem considered in this paper is to obtain a tight bound on the number of semi-algebraically connected components of the realizations of all realizable sign conditions of a family of polynomials $\mathcal{P} \subset \mathrm{R}\left[X_{1}, \ldots, X_{k}\right]$ in a variety $\operatorname{Zer}\left(\mathcal{Q}, \mathrm{R}^{k}\right)$ having dimension $k^{\prime} \leq k$, in terms of $s=\operatorname{card} \mathcal{P}, k, k^{\prime}$ and the degrees of the polynomials in $\mathcal{P}$ and $\mathcal{Q}$. 


\subsection{History and Prior Results}

The problem of bounding the number of semi-algebraically connected components (as well as the higher Betti numbers) has a long history. The initial results were obtained by Oleĭnik and Petrovskiu [20], and later by Thom [23] and Milnor [19], who proved a bound of $O(d)^{k}$ on the sum of the Betti numbers of any real algebraic variety in $\mathrm{R}^{k}$ defined by polynomials of degree at most $d$. This result has been generalized to arbitrary semi-algebraic sets in several different ways. The reader is referred to [8] for a survey of results in this direction and the references therein.

In [21], Pollack and Roy proved a bound of $\left(\begin{array}{l}s \\ k\end{array}\right) O(d)^{k}$ on the number of semialgebraically connected components of the realizations of all realizable sign conditions of a family of $s$ polynomials of degrees bounded by $d$. The proof was based on Oleĭnik-Petrovskiı̌-Thom-Milnor bounds for algebraic sets, as well as some deformation techniques and general position arguments. Similar results due to Alon [1] and Warren [26] only on the number of realizable sign conditions were known before.

It was soon realized that in some applications, notably in geometric transversal theory, as well as in bounding the complexity of the configuration space in robotics, it is useful to study the realizations of sign conditions of a family of $s$ polynomials in $\mathrm{R}\left[X_{1}, \ldots, X_{k}\right]$ restricted to a real variety $\operatorname{Zer}\left(Q, \mathrm{R}^{k}\right)$ where the real dimension of the variety $\operatorname{Zer}\left(Q, \mathrm{R}^{k}\right)$ can be much smaller than $k$. In [6], it was shown that the number of semi-algebraically connected components of the realizations of all realizable sign condition of a family, $\mathcal{P} \subset \mathrm{R}\left[X_{1}, \ldots, X_{k}\right]$ of $s$ polynomials, restricted to a real variety of dimension $k^{\prime}$, where the degrees of the polynomials in $\mathcal{P} \cup\{Q\}$ are all bounded by $d$, is bounded by $\left(\begin{array}{c}s \\ k^{\prime}\end{array}\right) O(d)^{k}$. This last result was made more precise in [5] where the authors bound (for each $i$ ) the sum of the $i$ th Betti number over all realizations of realizable sign conditions of a family of polynomials restricted to a variety of dimension $k^{\prime}$ by

$$
\sum_{0 \leq j \leq k^{\prime}-i}\left(\begin{array}{l}
s \\
j
\end{array}\right) 4^{j} d(2 d-1)^{k-1} .
$$

Notice that there is no undetermined constant in the above bound, and that it generalizes the bound in [6] which is the special case with $i=0$. The technique of the proof uses a generalization of the Mayer-Vietoris exact sequence in conjunction with the Oleĭnik-Petrovskiı̌-Thom-Milnor bounds on the Betti numbers of real varieties.

In a slightly different direction, an asymptotically tight bound (asymptotic with respect to the number of polynomials with fixed degrees and number of variables)

$$
\sum_{\sigma \in\{0,1,-1\}^{\mathcal{P}}} b_{0}\left(\sigma, \mathrm{R}^{k}\right) \leq \frac{(2 d)^{k}}{k !} s^{k}+O\left(s^{k-1}\right)
$$

was proved in [10]. This bound has the best possible leading term (as a function of $s$ ) but is not optimal with regards to the dependence on $d$, and thus is not directly comparable to the results in the current paper. 


\subsection{Main Result}

In this paper, we prove a bound on the number of semi-algebraically connected components over all realizable sign conditions of a family of polynomials in a variety. However, unlike in the above bound the role of the degrees of the polynomials defining the variety $V$ is distinguished from the role of the degrees of the polynomials in the family $\mathcal{P}$. This added flexibility seems to be necessary in certain applications of these bounds in combinatorial geometry (notably in the recent paper by Solymosi and Tao [22]). We give another application in the theory of geometric permutations in Sect. 4.

Our main result is the following theorem.

Theorem 1.1 Let $\mathrm{R}$ be a real closed field, and let $\mathcal{Q}, \mathcal{P} \subset \mathrm{R}\left[X_{1}, \ldots, X_{k}\right]$ be finite subsets of polynomials such that $\operatorname{deg}(Q) \leq d_{0}$ for all $Q \in \mathcal{Q}, \operatorname{deg} P=d_{P}$ for all $P \in \mathcal{P}$, and the real dimension of $\operatorname{Zer}\left(\mathcal{Q}, \mathrm{R}^{k}\right)$ is $k^{\prime} \leq k$. Suppose also that card $\mathcal{P}=s$, and for $\mathcal{I} \subseteq \mathcal{P}$ let $d_{\mathcal{I}}=\prod_{P \in \mathcal{I}} d_{P}$. Then,

$$
\sum_{\sigma \in\{0,1,-1\}^{\mathcal{P}}} b_{0}\left(\mathcal{R}\left(\sigma, \operatorname{Zer}\left(\mathcal{Q}, \mathrm{R}^{k}\right)\right)\right)
$$

is at most

$$
\sum_{\substack{\mathcal{I} \subset \mathcal{P} \\
\# \mathcal{I} \leq k^{\prime}}} 4^{\# \mathcal{I}}\left(\left(\begin{array}{c}
k+1 \\
k-k^{\prime}+\# \mathcal{I}+1
\end{array}\right)\left(2 d_{0}\right)^{k-k^{\prime}} d_{\mathcal{I}} \max _{P \in \mathcal{I}}\left\{2 d_{0}, d_{P}\right\}^{k^{\prime}-\# \mathcal{I}}+2(k-\# \mathcal{I}+1)\right) .
$$

In particular, if $d_{P} \leq d$ for all $P \in \mathcal{P}$, we have that

$$
\sum_{\sigma \in\{0,1,-1\}^{\mathcal{P}}} b_{0}\left(\mathcal{R}\left(\sigma, \operatorname{Zer}\left(\mathcal{Q}, \mathrm{R}^{k}\right)\right)\right)
$$

is at most

$$
\sum_{j=0}^{k^{\prime}} 4^{j}\left(\begin{array}{c}
s+1 \\
j
\end{array}\right)\left(\left(\begin{array}{c}
k+1 \\
k-k^{\prime}+j+1
\end{array}\right)\left(2 d_{0}\right)^{k-k^{\prime}} d^{j} \max \left\{2 d_{0}, d\right\}^{k^{\prime}-j}+2(k-j+1)\right) .
$$

\subsection{A Few Remarks}

Remark 1.2 The bound in Theorem 1.1 is tight (up to a factor of $O(1)^{k}$ ). It is instructive to examine the two extreme cases, when $k^{\prime}=0$ and $k^{\prime}=k-1$, respectively. When, $k^{\prime}=0$, the variety $\operatorname{Zer}\left(\mathcal{Q}, \mathrm{R}^{k}\right)$ is zero-dimensional, and is a union of at most $O\left(d_{0}\right)^{k}$ isolated points. The bound in Theorem 1.1 reduces to $O\left(d_{0}\right)^{k}$ in this case, and is thus tight.

When $k^{\prime}=k-1$ and $2 d_{0} \leq d$, the bound in Theorem 1.1 is equal to

$$
\sum_{j=0}^{k-1} 4^{j}\left(\begin{array}{c}
s+1 \\
j
\end{array}\right)\left(\left(\begin{array}{c}
k+1 \\
j+2
\end{array}\right) 2 d_{0} d^{j}+2(k-j+1)\right)=d_{0} O(s d)^{k-1} .
$$


The following example shows that this is the best possible (again up to $O(1)^{k}$ ).

Example 1.3 Let $\mathcal{P}$ be the set of $s$ polynomials in $X_{1}, \ldots, X_{k}$ each of which is a product of $d$ generic linear forms. Let $\mathcal{Q}=\{Q\}$, where

$$
Q=\prod_{1 \leq i \leq d_{0}}\left(X_{k}-i\right) .
$$

It is easy to see that in this case the number of semi-algebraically connected components of all realizable strict sign conditions of $\mathcal{P}$ (i.e., sign conditions belonging to $\left.\{-1,+1\}^{\mathcal{P}}\right)$ on $\operatorname{Zer}\left(\mathcal{Q}, \mathrm{R}^{k}\right)$ is equal to

$$
d_{0} \sum_{i=0}^{k-1}\left(\begin{array}{c}
s d \\
i
\end{array}\right)=d_{0}(\Omega(s d))^{k-1},
$$

since the intersection of $\bigcup_{P \in \mathcal{P}} \operatorname{Zer}\left(P, \mathrm{R}^{k}\right)$ with the hyperplane defined by $X_{k}=i$ for each $i, i=1, \ldots, d_{0}$, is homeomorphic to an union of $s d$ generic hyperplanes in $\mathrm{R}^{k-1}$, and the number of connected components of the complement of the union of $s d$ generic hyperplanes in $\mathrm{R}^{k-1}$ is precisely $\sum_{i=0}^{k-1}\left(\begin{array}{c}s d \\ i\end{array}\right)$.

Remark 1.4 Most bounds on the number of semi-algebraically connected components of real algebraic varieties are stated in terms of the maximum of the degrees of the polynomials defining the variety (rather than in terms of the degree sequence). One reason behind this is the well-known fact that a "Bezout type" theorem is not true for real algebraic varieties. The number of semi-algebraically connected components (indeed even isolated zeros) of a set of polynomials $\left\{P_{1}, \ldots, P_{m}\right\} \subset$ $\mathrm{R}\left[X_{1}, \ldots, X_{k}, X_{k+1}\right]$ with degrees $d_{1}, \ldots, d_{m}$ can be greater than the product $d_{1} \cdots d_{m}$, as can be seen in the following example.

Example 1.5 Let $\mathcal{P}=\left\{P_{1}, \ldots, P_{m}\right\} \subset \mathrm{R}\left[X_{1}, \ldots, X_{k+1}\right]$ be defined as follows:

$$
\begin{aligned}
P_{1} & =\sum_{i=1}^{k} \prod_{j=1}^{d}\left(X_{i}-j\right)^{2}, \\
P_{j} & =\prod_{i=1}^{m-j+2}\left(X_{k+1}-i\right), \quad 2 \leq j \leq m .
\end{aligned}
$$

Let $\mathcal{P}_{i}=\left\{P_{1}, \ldots, P_{i}\right\}$. Notice that for each $i, 1 \leq i<m, \operatorname{Zer}\left(\mathcal{P}_{i}, \mathrm{R}^{k+1}\right)$ strictly contains $\operatorname{Zer}\left(\mathcal{P}_{i+1}, \mathrm{R}^{k+1}\right)$. Moreover, $b_{0}\left(\operatorname{Zer}\left(\mathcal{P}, \mathrm{R}^{k+1}\right)\right)=2 d^{k}$, while the product of the degrees of the polynomials in $\mathcal{P}$ is $2 d m$ !. Clearly, for $d$ large enough $2 d^{k}>2 d m$ !.

Remark 1.6 Most of the previously known bounds on the Betti numbers of realizations of sign conditions relied ultimately on the Oleĭnik-Petrovskiŭ-Thom-Milnor bounds on the Betti numbers of real varieties. Since in the proofs of these bounds the finite family of polynomials defining a given real variety is replaced by a single 
polynomial by taking a sum of squares, it is not possible to separate out the different roles played by the degrees of the polynomials in $\mathcal{P}$ and those in $\mathcal{Q}$. The technique used in this paper avoids using the Oleĭnik-Petrovskiū-Thom-Milnor bounds, but uses directly classically known formulas for the Betti numbers of smooth, complete intersections in complex projective space. The bounds obtained from these formulas depend more delicately on the individual degrees of the polynomials involved (see Corollary 2.3), and this allows us to separate the roles of $d$ and $d_{0}$ in our proof.

\subsection{Outline of the Proof of Theorem 1.1}

The main idea behind our improved bound is to reduce the problem of bounding the number of semi-algebraically connected components of all sign conditions on a variety to the problem of bounding the sum of the $\mathbb{Z}_{2}$-Betti numbers of certain smooth complete intersections in complex projective space. This is done as follows. First, assume that $\operatorname{Zer}\left(\mathcal{Q}, \mathrm{R}^{k}\right)$ is bounded. The general case is reduced to this case by an initial step using the conical triviality of semi-algebraic sets at infinity.

Assuming that $\operatorname{Zer}\left(\mathcal{Q}, \mathrm{R}^{k}\right)$ is bounded, and letting $Q=\sum_{F \in \mathcal{Q}} F^{2}$, we consider another polynomial $\operatorname{Def}(Q, H, \zeta)$ which is an infinitesimal perturbation of $Q$. The basic semi-algebraic set, $T$, defined by $\operatorname{Def}(Q, H, \zeta) \leq 0$ is a semi-algebraic subset of $\mathrm{R}\langle\zeta\rangle^{k}$ (where $\mathrm{R}\langle\zeta\rangle$ is the field of algebraic Puiseux series with coefficients in $\mathrm{R}$, see Sect. 2.5 below for properties of the field of Puiseux series that we need in this paper). The semi-algebraic set $T$ has the property that for each semi-algebraically connected component $C$ of $\operatorname{Zer}\left(Q, \mathrm{R}^{k}\right)$ there exists a semi-algebraically connected component $D$ of $T$, which is bounded over R and such that $\lim _{\zeta} D=C$ (see Sect. 2.5 for definition of $\lim _{\zeta}$ ). The semi-algebraic set $T$ should be thought of as an infinitesimal "tube" around $\operatorname{Zer}\left(Q, \mathrm{R}^{k}\right)$, which is bounded by a smooth hypersurface (namely, $\left.\operatorname{Zer}\left(\operatorname{Def}(Q, H, \zeta), \mathrm{R}\langle\zeta\rangle^{k}\right)\right)$. We then show it is possible to cut out a $k^{\prime}$-dimensional subvariety, $W$ in $\operatorname{Zer}\left(\operatorname{Def}(Q, H, \zeta), \mathrm{R}\langle\zeta\rangle^{k}\right)$, such that (for generic choice of coordinates) in fact $\lim _{\zeta} W=\operatorname{Zer}\left(Q, \mathrm{R}^{k}\right.$ ) (Proposition 3.4), and moreover the homogenizations of the polynomials defining $W$ define a non-singular complete intersection in $\mathbb{P}_{\mathrm{C}\langle\zeta\rangle}^{k}$ (Proposition 3.6). $W$ is defined by $k-k^{\prime}$ forms of degree at most $2 d_{0}$. In order to bound the number of semi-algebraically connected components of realizations of sign conditions of the family $\mathcal{P}$ on $\operatorname{Zer}\left(Q, \mathrm{R}^{k}\right)$, we need to bound the number of semi-algebraically connected components of the intersection of $W$ with the zeros of certain infinitesimal perturbations of polynomials in $\mathcal{P}$ (see Proposition 3.9 below). The number of cases that we need to consider is bounded by $\left(\begin{array}{c}O(s) \\ k^{\prime}\end{array}\right)$, and again each such set of polynomials define a non-singular complete intersection of $p, k-k^{\prime} \leq p \leq k$ hypersurfaces in $k$-dimensional projective space over an appropriate algebraically closed field, $k-k^{\prime}$ of which are defined by forms having degree at most $2 d_{0}$ and the remaining $m=p-k+k^{\prime}$ of degree bounded by $d$. In this situation, there are classical formulas known for the Betti numbers of such varieties, and they imply a bound of $\left(\begin{array}{c}k+1 \\ m+1\end{array}\right)\left(2 d_{0}\right)^{k-k^{\prime}} d^{k^{\prime}}+O(k)$ on the sum of the Betti numbers of such varieties (see Corollary 2.3 below). The bounds on the sum of the Betti numbers of these projective complete intersections in the algebraic closure imply using the well-known Smith inequality (see Theorem 2.4) a bound on the number of semi-algebraically connected components of the real parts of these varieties, and 
in particular the number of bounded components. The product of the two bounds, namely the combinatorial bound on the number of different cases and the algebraic part depending on the degrees, summed appropriately lead to the claimed bound.

\subsection{Connection to Prior Work}

The idea of approximating an arbitrary real variety of dimension $k^{\prime}$ by a complete intersection was used in [7] to give an efficient algorithm for computing sample points in each semi-algebraically connected component of all realizable sign conditions of a family of polynomials restricted to the variety. Because of complexity issues related to algorithmically choosing a generic system of co-ordinates however, instead of choosing a single generic system of co-ordinates, a finite universal family of different co-ordinate systems was used to approximate the variety. Since in this paper we are not dealing with algorithmic complexity issues, we are free to choose generic coordinates. Note also that the idea of bounding the number of semi-algebraically connected components of realizable sign conditions or of real algebraic varieties, using known formulas for Betti numbers of non-singular, complete intersections in complex projective spaces, and then using Smith inequality, have been used before in several different settings (see [4] in the case of semi-algebraic sets defined by quadrics and [11] for arbitrary real algebraic varieties).

The rest of the paper is organized as follows. In Sect. 2, we state some known results that we will need to prove the main theorem. These include explicit recursive formulas for the sum of Betti numbers of non-singular, complete intersections of complex projective varieties (Sect. 2.1), the Smith inequality relating the Betti numbers of complex varieties defined over $\mathrm{R}$ with those of their real parts (Sect. 2.2), some results about generic choice of coordinates (Sects. 2.4, 2.3), and finally a few facts about non-archimedean extensions and Puiseux series that we need for making perturbations (Sect. 2.5). We prove the main theorem in Sect. 3.

\section{Certain Preliminaries}

2.1 The Betti Numbers of a Non-Singular Complete Intersection in Complex Projective Space

If $\mathcal{P}$ is a finite subset of $\mathrm{R}\left[X_{1}, \ldots, X_{k}\right]$ consisting of homogeneous polynomials we denote the set of zeros of $\mathcal{P}$ in $\mathbb{P}_{\mathrm{R}}^{k}$ (resp., in $\mathbb{P}_{\mathrm{C}}^{k}$ ) by

$$
\begin{aligned}
\operatorname{Zer}\left(\mathcal{P}, \mathbb{P}_{\mathrm{R}}^{k}\right) & =\left\{x \in \mathbb{P}_{\mathrm{R}}^{k} \mid \bigwedge_{P \in \mathcal{P}} P(x)=0\right\} \\
\left(\text { resp., } \operatorname{Zer}\left(\mathcal{P}, \mathbb{P}_{\mathrm{C}}^{k}\right)\right. & \left.=\left\{x \in \mathbb{P}_{\mathrm{C}}^{k} \mid \bigwedge_{P \in \mathcal{P}} P(x)=0\right\}\right) .
\end{aligned}
$$

For $P \in \mathrm{R}\left[X_{1}, \ldots, X_{k}\right]$ we will denote by $\operatorname{Zer}\left(P, \mathrm{R}^{k}\right)\left(\operatorname{resp} ., \operatorname{Zer}\left(P, \mathrm{C}^{k}\right), \operatorname{Zer}\left(P, \mathbb{P}_{\mathrm{R}}^{k}\right)\right.$, $\left.\operatorname{Zer}\left(P, \mathbb{P}_{\mathrm{C}}^{k}\right)\right)$ the variety $\operatorname{Zer}\left(\{P\}, \mathrm{R}^{k}\right) \quad\left(\right.$ resp., $\operatorname{Zer}\left(\{P\}, \mathrm{C}^{k}\right), \quad \operatorname{Zer}\left(\{P\}, \mathbb{P}_{\mathrm{R}}^{k}\right)$, $\left.\operatorname{Zer}\left(\{P\}, \mathbb{P}_{\mathrm{C}}^{k}\right)\right)$. 
For any locally closed semi-algebraic set $X$, we denote by $b_{i}(X)$ the dimension of

$$
\mathrm{H}_{i}\left(X, \mathbb{Z}_{2}\right)
$$

the $i$ th homology group of $X$ with coefficients in $\mathbb{Z}_{2}$. We refer to [9, Chap. 6] for the definition of homology groups in case the field $\mathrm{R}$ is not the field of real numbers. Note that $b_{0}(X)$ equals the number of semi-algebraically connected components of the semi-algebraic set $X$.

For $\sigma \in\{0,1,-1\}^{\mathcal{P}}$ and $V \subset \mathrm{R}^{k}$ a closed semi-algebraic set, we will denote by $b_{i}(\sigma, V)$ the dimension of

$$
\mathrm{H}_{i}\left(\mathcal{R}(\sigma, V), \mathbb{Z}_{2}\right)
$$

We will denote by

$$
b(\sigma, V)=\sum_{i \geq 0} b_{i}(\sigma, V)
$$

Definition 2.1 A projective variety $X \subset \mathbb{P}_{\mathrm{C}}^{k}$ of codimension $n$ is a non-singular complete intersection if it is the intersection of $n$ non-singular hypersurfaces in $\mathbb{P}_{\mathrm{C}}^{k}$ that meet transversally at each point of the intersection.

Fix an $m$-tuple of natural numbers $\bar{d}=\left(d_{1}, \ldots, d_{m}\right)$. Let $X_{\mathrm{C}}=\operatorname{Zer}\left(\left\{Q_{1}, \ldots, Q_{m}\right\}\right.$, $\mathbb{P}_{\mathrm{C}}^{k}$ ), such that the degree of $Q_{i}$ is $d_{i}$, denote a complex projective variety of codimension $m$ which is a non-singular complete intersection. It is a classical fact that the Betti numbers of $X_{\mathrm{C}}$ depend only on the degree sequence and not on the specific $X_{\mathrm{C}}$. In fact, it follows from Lefshetz theorem on hyperplane sections (see, for example, $[25$, Sect. 1.2.2]) that

$$
b_{i}\left(X_{\mathrm{C}}\right)=b_{i}\left(\mathbb{P}_{\mathrm{C}}^{k}\right), \quad 0 \leq i<k-m .
$$

Also, by Poincaré duality we have that

$$
b_{i}\left(X_{\mathrm{C}}\right)=b_{2(k-m)-i}\left(X_{\mathrm{C}}\right), \quad 0 \leq i \leq k-m .
$$

Thus, all the Betti numbers of $X_{\mathrm{C}}$ are determined once we know $b_{k-m}\left(X_{\mathrm{C}}\right)$, or equivalently the Euler-Poincaré characteristic

$$
\chi\left(X_{\mathrm{C}}\right)=\sum_{i \geq 0}(-1)^{i} b_{i}\left(X_{\mathrm{C}}\right)
$$

Denoting $\chi\left(X_{\mathrm{C}}\right)$ by $\chi_{m}^{k}\left(d_{1}, \ldots, d_{m}\right)$ (since it only depends on the degree sequence) we have the following recurrence relation (see, for example, [11]).

$$
\begin{aligned}
& \chi_{m}^{k}\left(d_{1}, \ldots, d_{m}\right) \\
& \quad= \begin{cases}k+1 & \text { if } m=0, \\
d_{1} \cdots d_{m} & \text { if } m=k, \\
d_{m} \chi_{m-1}^{k-1}\left(d_{1}, \ldots, d_{m-1}\right)-\left(d_{m}-1\right) \chi_{m}^{k-1}\left(d_{1}, \ldots, d_{m}\right) & \text { if } 0<m<k\end{cases}
\end{aligned}
$$


We have the following inequality.

Proposition 2.2 Suppose $1 \leq d_{1} \leq d_{2} \leq \cdots \leq d_{m}$. The function $\chi_{m}^{k}\left(d_{1}, \ldots, d_{m}\right)$ satisfies

$$
\left|\chi_{m}^{k}\left(d_{1}, \ldots, d_{m}\right)\right| \leq\left(\begin{array}{c}
k+1 \\
m+1
\end{array}\right) d_{1} \cdots d_{m-1} d_{m}^{k-m+1} .
$$

Proof The proof is by induction in each of the three cases of (2.1).

Case $m=0$ :

$$
\chi_{0}^{k}=k+1 \leq\left(\begin{array}{c}
k+1 \\
1
\end{array}\right)=k+1
$$

Case $m=k$ :

$$
\chi_{m}^{k}\left(d_{1}, \ldots, d_{m}\right)=d_{1} \cdots d_{m-1} d_{m} \leq\left(\begin{array}{c}
k+1 \\
k+1
\end{array}\right) d_{1} \cdots d_{m-1} d_{m}=d_{1} \cdots d_{m-1} d_{m} .
$$

Case $0<m<k$ :

$$
\begin{aligned}
& \left|\chi_{m}^{k}\left(d_{1}, \ldots, d_{m}\right)\right| \\
& =\left|d_{m} \chi_{m-1}^{k-1}\left(d_{1}, \ldots, d_{m-1}\right)-\left(d_{m}-1\right) \chi_{m}^{k-1}\left(d_{1}, \ldots, d_{m}\right)\right| \\
& \leq d_{m}\left|\chi_{m-1}^{k-1}\left(d_{1}, \ldots, d_{m-1}\right)\right|+d_{m}\left|\chi_{m}^{k-1}\left(d_{1}, \ldots, d_{m}\right)\right| \\
& \leq d_{m}\left(\begin{array}{c}
k \\
m
\end{array}\right) d_{1} \cdots d_{m-2} d_{m-1}^{(k-1)-(m-1)+1}+d_{m}\left(\begin{array}{c}
k \\
m+1
\end{array}\right) d_{1} \cdots d_{m-1} d_{m}^{(k-1)-m+1} \\
& \stackrel{*}{\leq}\left(\begin{array}{c}
k \\
m
\end{array}\right) d_{1} \cdots d_{m-1} d_{m}^{k-m+1}+\left(\begin{array}{c}
k \\
m+1
\end{array}\right) d_{1} \cdots d_{m-1} d_{m}^{k-m+1} \\
& =\left(\begin{array}{c}
k+1 \\
m+1
\end{array}\right) d_{1} \cdots d_{m-1} d_{m}^{k-m+1},
\end{aligned}
$$

where the inequality $\stackrel{*}{\leq}$ follows from the observation

$$
d_{m-1}^{(k-1)-(m-1)+1} \leq d_{m-1} d_{m}^{k-m},
$$

since $d_{m-1} \leq d_{m}$ by assumption, and the last equality is from the identity $\left(\begin{array}{c}k+1 \\ m+1\end{array}\right)=$ $\left(\begin{array}{l}k \\ m\end{array}\right)+\left(\begin{array}{c}k \\ m+1\end{array}\right)$.

Now let $\beta_{m}^{k}\left(d_{1}, \ldots, d_{m}\right)$ denote $\sum_{i \geq 0} b_{i}\left(X_{\mathrm{C}}\right)$.

The following corollary is an immediate consequence of Proposition 2.2 and the remarks preceding it.

\section{Corollary 2.3}

$$
\beta_{m}^{k}\left(d_{1}, \ldots, d_{m}\right) \leq\left(\begin{array}{c}
k+1 \\
m+1
\end{array}\right) d_{1} \cdots d_{m-1} d_{m}^{k-m+1}+2(k-m+1) .
$$




\subsection{Smith Inequality}

We state a version of the Smith inequality which plays a crucial role in the proof of the main theorem. Recall that for any compact topological space equipped with an involution, inequalities derived from the Smith exact sequence allows one to bound the sum of the Betti numbers (with $\mathbb{Z}_{2}$ coefficients) of the fixed point set of the involution by the sum of the Betti numbers (again with $\mathbb{Z}_{2}$ coefficients) of the space itself (see, for instance, [24, p. 131]). In particular, we have for a complex projective variety defined by real forms, with the involution taken to be complex conjugation, the following theorem.

Theorem 2.4 (Smith inequality) Let $\mathcal{Q} \subset \mathrm{R}\left[X_{1}, \ldots, X_{k+1}\right]$ be a family of homogeneous polynomials. Then,

$$
b\left(\operatorname{Zer}\left(\mathcal{Q}, \mathbb{P}_{\mathrm{R}}^{k}\right)\right) \leq b\left(\operatorname{Zer}\left(\mathcal{Q}, \mathbb{P}_{\mathrm{C}}^{k}\right)\right)
$$

Remark 2.5 Note that we are going to use Theorem 2.4 only for bounding the number of semi-algebraically connected components (that is, the zeroth Betti number) of certain real varieties. Nevertheless, to apply the inequality we need a bound on the sum of all the Betti numbers (not just $b_{0}$ ) on the right-hand side.

The following theorem used in the proof of Theorem 1.1 is a direct consequence of Theorem 2.4 and the bound in Corollary 2.3.

Theorem 2.6 Let $\mathrm{R}$ be a real closed field and $\mathcal{P}=\left\{P_{1}, \ldots, P_{m}\right\} \subset \mathrm{R}\left[X_{1}, \ldots, X_{k}\right]$ with $\operatorname{deg}\left(P_{i}\right)=d_{i}, i=1, \ldots, m$, and $1 \leq d_{1} \leq d_{2} \leq \cdots \leq d_{m}$. Let $\mathcal{P}^{h}=$ $\left\{P_{1}^{h}, \ldots, P_{m}^{h}\right\}$ be the corresponding set of homogenized polynomials, and suppose that $P_{1}^{h}, \ldots, P_{m}^{h}$ define a non-singular complete intersection in $\mathbb{P}_{\mathrm{C}}^{k}$. Then,

$$
b_{0}\left(\operatorname{Zer}\left(\mathcal{P}^{h}, \mathbb{P}_{\mathrm{R}}^{k}\right)\right) \leq\left(\begin{array}{c}
k+1 \\
m+1
\end{array}\right) d_{1} \cdots d_{m-1} d_{m}^{k-m+1}+2(k-m+1) .
$$

In case $\operatorname{Zer}\left(\mathcal{P}, \mathrm{R}^{k}\right)$ is bounded,

$$
b_{0}\left(\operatorname{Zer}\left(\mathcal{P}, \mathrm{R}^{k}\right)\right) \leq\left(\begin{array}{c}
k+1 \\
m+1
\end{array}\right) d_{1} \cdots d_{m-1} d_{m}^{k-m+1}+2(k-m+1) .
$$

Proof Proof is immediate from Theorem 2.4 and Corollary 2.3.

Remark 2.7 Note that the bound in Theorem 2.6 is not true if we omit the assumption of being a non-singular complete intersection. A counter-example is provided by Example 1.5.

Remark 2.8 Another possible approach to the proof of Theorem 2.6 is to use the critical point method and bound directly the number of critical points of a generic projection using the multi-homogeneous Bézout theorem (see, for example, [14]). 


\subsection{Generic Coordinates}

Unless otherwise stated, for any real closed field R, we are going to use the Euclidean topology (see, for example, [12, p. 26]) on $\mathrm{R}^{k}$. Sometimes we will need to use (the coarser) Zariski topology, and we explicitly state this whenever it is the case.

Notation 2.9 For a real algebraic set $V=\operatorname{Zer}\left(Q, \mathrm{R}^{k}\right)$ we let $\mathrm{reg} V$ denote the nonsingular points in dimension $\operatorname{dim} V$ of $V$ [12, Definition 3.3.9].

Definition 2.10 Let $V=\operatorname{Zer}\left(Q, \mathrm{R}^{k}\right)$ be a real algebraic set. Define $V^{(k)}=V$, and for $0 \leq i \leq k-1$ define

$$
V^{(i)}=V^{(i+1)} \backslash \operatorname{reg} V^{(i+1)} .
$$

Set $\operatorname{dim} V^{(i)}=d(i)$

Remark 2.11 Note that $V^{(i)}$ is Zariski-closed for each $0 \leq i \leq k$.

Notation 2.12 We denote by $\operatorname{Gr}_{\mathrm{R}}(k, j)$ the real Grassmannian of $j$-dimensional linear subspaces of $\mathrm{R}^{k}$.

Notation 2.13 For a real algebraic variety $V \subset \mathrm{R}^{k}$, and $x \in \operatorname{reg} V$ where $\operatorname{dim} r e g=p$, we denote by $T_{x} V$ the tangent space at $x$ to $V$ (translated to the origin). Note that $T_{x} V$ is a p-dimensional subspace of $\mathrm{R}^{k}$, and hence an element of $\operatorname{Gr}_{\mathrm{R}}(k, p)$.

Definition 2.14 Let $V=\operatorname{Zer}\left(Q, \mathrm{R}^{k}\right)$ be a real algebraic set, $1 \leq j \leq k$, and $\ell \in$ $\operatorname{Gr}(k, k-j)$. We say the linear space $\ell$ is $j$-good with respect to $V$ if either:

1. $j \notin d([0, k])$, or

2. $d(i)=j$, and

$$
A_{\ell}:=\left\{x \in \operatorname{reg} V^{(i)} \mid \operatorname{dim}\left(\mathrm{T}_{x} V^{(i)} \cap \ell\right)=0\right\}
$$

is a nonempty dense Zariski-open subset of reg $V^{(i)}$.

Remark 2.15 Note that the semi-algebraic subset $A_{\ell}$ is always a (possibly empty) Zariski-open subset of reg $V^{(i)}$, hence of $V^{(i)}$. In the case where $V^{(i)}$ is an irreducible Zariski-closed subset (see Remark 2.11), the set $A_{\ell}$ is either empty or a nonempty dense Zariski-open subset of reg $V^{(i)}$.

Definition 2.16 Let $V=\operatorname{Zer}\left(Q, \mathrm{R}^{k}\right)$ be a real algebraic set and $\mathcal{B}=\left\{v_{1}, \ldots, v_{k}\right\} \subset$ $\mathrm{R}^{k}$ a basis of $\mathrm{R}^{k}$. We say that the basis $\mathcal{B}$ is good with respect to $V$ if for each $j$, $1 \leq j \leq k$, the linear space $\operatorname{span}\left\{v_{1}, \ldots, v_{k-j}\right\}$ is $j$-good.

Proposition 2.17 Let $V=\operatorname{Zer}\left(Q, \mathrm{R}^{k}\right)$ be a real algebraic set and $\left\{v_{1}, \ldots, v_{k}\right\} \subset$ $\mathrm{R}^{k}$ a basis of $\mathrm{R}^{k}$. Then, there exists a nonempty open semi-algebraic subset of linear transformations $\mathcal{O} \subset \mathrm{GL}(k, \mathrm{R})$ such that for every $T \in \mathcal{O}$ the basis $\left\{T\left(v_{1}\right), \ldots, T\left(v_{k}\right)\right\}$ is good with respect to $V$. 
The proof of Proposition 2.17 uses the following notation and lemma.

Notation 2.18 For any $\ell \in \mathrm{Gr}_{\mathrm{R}}(k, k-j), 1 \leq j \leq k$, we denote by $\Omega(\ell)$ the real algebraic subvariety of $\mathrm{Gr}_{\mathrm{R}}(k, j)$ defined by

$$
\Omega(\ell)=\left\{\ell^{\prime} \in \operatorname{Gr}_{\mathrm{R}}(k, j) \mid \ell \cap \ell^{\prime} \neq 0\right\} .
$$

Lemma 2.19 For any nonempty open semi-algebraic subset $U \subset \operatorname{Gr}_{\mathrm{R}}(k, k-j), 1 \leq$ $j \leq k$, we have

$$
\bigcap_{\ell \in U} \Omega(\ell)=\emptyset .
$$

Proof We use a technique due to Chistov et al. (originally appearing in [13]) who explicitly constructed a finite family of elements in $\operatorname{Gr}_{\mathrm{R}}(k, k-j)$ such that every $\ell^{\prime} \in \mathrm{Gr}_{\mathrm{R}}(k, j)$ is transversal to at least one member of this family. More precisely, let $e_{0}, \ldots, e_{k-1}$ be the standard basis vectors in $\mathrm{R}^{k}$, and let for any $x \in \mathrm{R}$,

$$
v_{k}(x)=\sum_{i=0}^{k-1} x^{i} e_{i}
$$

Then the set of vectors $v_{k}(x), v_{k}(x+1), \ldots, v_{k}(x+k-j-1)$ are linearly independent and span a $(k-j)$-dimensional subspace of $\mathrm{R}^{k}$. Denote by $\ell_{x}$ the corresponding element in $\operatorname{Gr}_{\mathrm{R}}(k, k-j)$. An easy adaptation of the proof of Proposition 13.27 [9] now shows that, for $\varepsilon>0$, the set

$$
L_{\varepsilon, k, j}:=\left\{\ell_{m \varepsilon} \mid 0 \leq m \leq k(k-j)\right\} \subset \operatorname{Gr}(k, k-j)
$$

has the property that, for any $\ell^{\prime} \in \mathrm{Gr}_{\mathrm{R}}(k, j)$, there exists some $m, 0 \leq m \leq k(k-j)$, such that $\ell^{\prime} \cap \ell_{m \varepsilon}=0$. In other words, for every $\varepsilon>0$,

$$
\bigcap_{0 \leq m \leq k(k-j)} \Omega\left(\ell_{m \varepsilon}\right)=\emptyset .
$$

By rotating coordinates, we can assume that $\ell_{0} \in U$, and then by choosing $\varepsilon$ small enough we can assume that $L_{\varepsilon, k, j} \subset U$. This finishes the proof.

Proof of Proposition 2.17 We prove that for each $j, 0 \leq j \leq k$, the set of $\ell \in$ $\operatorname{Gr}_{\mathrm{R}}(k, k-j)$ such that $\ell$ is not $j$-good for $V$ is a semi-algebraic subset of $\operatorname{Gr}_{\mathrm{R}}(k, k-j)$ without interior. It then follows that its complement contains an open dense semi-algebraic subset of $\operatorname{Gr}_{\mathrm{R}}(k, k-j)$, and hence there is an open semi-algebraic subset $\mathcal{O}_{j} \subset \mathrm{GL}_{n}(\mathrm{R})$ such that for each $T \in \mathcal{O}_{j}$, the linear space $\operatorname{span}\left\{T\left(v_{1}\right), \ldots, T\left(v_{k-j}\right)\right\}$ is $j$-good with respect to $V$.

Let $j=d(i), 0 \leq i \leq k$. Seeking a contradiction, suppose that there is an open semi-algebraic subset $U \subset \operatorname{Gr}_{\mathrm{R}}(k, k-j)$ such that every $\ell \in U$ is not $j$-good with respect to $V$. Let $V_{1}^{(i)}, \ldots, V_{n}^{(i)}$ be the distinct irreducible components of the Zariskiclosed set $V^{(i)}$. For each $\ell \in U, \ell$ is not $j$-good for some $V_{r}^{(i)}, 1 \leq r \leq n$ (otherwise 
$\ell$ would be $j$-good for $V$ ). Let $U_{1}, \ldots, U_{n}$ denote the semi-algebraic sets defined by

$$
U_{r}:=\left\{\ell \in U \mid \ell \text { is not } j \text {-good for } V_{r}^{(i)}\right\} .
$$

We have $U=U_{1} \cup \cdots \cup U_{n}$, and $U$ is open in $\operatorname{Gr}_{\mathrm{R}}(k, k-j)$. Hence, for some $r$, $1 \leq r \leq n$, we have $U_{r}$ contains an non-empty open semi-algebraic subset. Replacing $U$ by this (possibly smaller) subset we have that the set $A_{\ell} \cap \operatorname{reg} V_{r}^{(i)}$ is empty for each $\ell \in U$ (cf. Definition 2.14, Remark 2.15). So, reg $V_{r}^{(i)} \subset \operatorname{reg} V^{(i)} \backslash A_{\ell}$ for every $\ell \in U$, and

$$
\emptyset \neq \operatorname{reg} V_{r}^{(i)} \subset \bigcap_{\ell \in U} \operatorname{reg} V^{(i)} \backslash A_{\ell} .
$$

Let $z \in \bigcap_{\ell \in U} \operatorname{reg} V^{(i)} \backslash A_{\ell}$, but then the linear space $\ell^{\prime}=T_{z}\left(\operatorname{reg} V^{(i)}\right)$ is in $\bigcap_{\ell \in U} \Omega(\ell)$, contradicting Lemma 2.19.

2.4 Non-Singularity of the Set Critical of Points of Hypersurfaces for Generic Projections

Notation 2.20 Let $H \in \mathrm{R}\left[X_{1}, \ldots, X_{k}\right]$. For $0 \leq p \leq k$, we will denote by $\operatorname{Cr}_{p}(H)$ the set of polynomials

$$
\left\{H, \frac{\partial H}{\partial X_{1}}, \ldots, \frac{\partial H}{\partial X_{p}}\right\} .
$$

We will denote by $\mathrm{Cr}_{p}^{h}(H)$ the corresponding set

$$
\left\{H^{h}, \frac{\partial H^{h}}{\partial X_{1}}, \ldots, \frac{\partial H^{h}}{\partial X_{p}}\right\}
$$

of homogenized polynomials.

Notation 2.21 Let $d$ be even. We will denote by $\operatorname{Pos}_{\mathrm{R}, d, k} \subset \mathrm{R}\left[X_{1}, \ldots, X_{k}\right]$ the set of non-negative polynomials in $\mathrm{R}\left[X_{1}, \ldots, X_{k}\right]$ of degree at most $d$. Denoting by $\mathrm{R}\left[X_{1}, \ldots, X_{k}\right]_{\leq d}$ the finite dimensional vector subspace of $\mathrm{R}\left[X_{1}, \ldots, X_{k}\right]$ consisting of polynomials of degree at most $d$, we have that $\operatorname{Pos}_{\mathrm{R}, d, k}$ is a (semi-algebraic) cone in $\mathrm{R}\left[X_{1}, \ldots, X_{k}\right]_{\leq d}$ with non-empty interior.

Proposition 2.22 Let $\mathrm{R}$ be a real closed field and $\mathrm{C}$ the algebraic closure of $\mathrm{R}$. Let $d>0$ be even. Then there exists $H \in \operatorname{Pos}_{\mathrm{R}, d, k}$, such that for each $p, 0 \leq p \leq k$, $\mathrm{Cr}_{p}^{h}(H)$ defines a non-singular complete intersection in $\mathbb{P}_{\mathrm{C}}^{k}$.

Proof The proposition follows from the fact that the generic polar varieties of nonsingular complex hypersurfaces are non-singular complete intersections [2, Proposition 3], and since $\operatorname{Pos}_{\mathrm{R}, d, k}$ has non-empty interior, we can choose a generic polynomial in $\operatorname{Pos}_{\mathrm{R}, d, k}$ having this property.

Remark 2.23 The fact that generic polar varieties of a non-singular complex variety are non-singular complete intersections is not true in general for higher codimension varieties, see [2, 3], in particular [3, Sect. 3]. 


\subsection{Infinitesimals and Puiseux Series}

In our arguments, we are going to use infinitesimals and non-archimedean extensions of a given real closed field R. A typical non-archimedean extension of $\mathrm{R}$ is the field $\mathrm{R}\langle\varepsilon\rangle$ of algebraic Puiseux series with coefficients in $\mathrm{R}$, which coincide with the germs of semi-algebraic continuous functions (see [9], Chap. 2, Sect. 6 and Chap. 3, Sect. 3). An element $x \in \mathrm{R}\langle\varepsilon\rangle$ is bounded over $\mathrm{R}$ if $|x| \leq r$ for some $0 \leq r \in \mathrm{R}$. The subring $\mathrm{R}\langle\varepsilon\rangle_{b}$ of elements of $\mathrm{R}\langle\varepsilon\rangle$ bounded over $\mathrm{R}$ consists of the Puiseux series with nonnegative exponents. We denote by $\lim _{\varepsilon}$ the ring homomorphism from $\mathrm{R}\langle\varepsilon\rangle_{b}$ to $\mathrm{R}$ which maps $\sum_{i \in \mathbb{N}} a_{i} \varepsilon^{i / q}$ to $a_{0}$. So, the mapping $\lim _{\varepsilon}$ simply replaces $\varepsilon$ by 0 in a bounded Puiseux series. Given $S \subset \mathrm{R}\langle\varepsilon\rangle^{k}$, we denote by $\lim _{\varepsilon}(S) \subset \mathrm{R}^{k}$ the image by $\lim _{\varepsilon}$ of the elements of $S$ whose coordinates are bounded over R. We denote by $\mathrm{R}\left\langle\varepsilon_{1}, \varepsilon_{2}, \ldots, \varepsilon_{\ell}\right\rangle$ the real closed field $\mathrm{R}\left\langle\varepsilon_{1}\right\rangle\left\langle\varepsilon_{2}\right\rangle \cdots\left\langle\varepsilon_{\ell}\right\rangle$, and we let $\lim _{\varepsilon_{i}}$ denote the ring homomorphism $\lim _{\varepsilon_{i}} \lim _{\varepsilon_{i+1}} \cdots \lim _{\varepsilon_{\ell}}$.

More generally, let $\mathrm{R}^{\prime}$ be a real closed field extension of $\mathrm{R}$. If $S \subset \mathrm{R}^{k}$ is a semialgebraic set, defined by a boolean formula $\Phi$ with coefficients in R, we denote by $\operatorname{Ext}\left(S, \mathrm{R}^{\prime}\right)$ the extension of $S$ to $\mathrm{R}^{\prime}$, i.e., the semi-algebraic subset of $\mathrm{R}^{\prime k}$ defined by $\Phi$. The first property of $\operatorname{Ext}\left(S, \mathrm{R}^{\prime}\right)$ is that it is well defined, i.e., independent on the formula $\Phi$ describing $S$ [9, Proposition 2.87]. Many properties of $S$ can be transferred to $\operatorname{Ext}\left(S, \mathrm{R}^{\prime}\right)$ : for example, $S$ is non-empty if and only if $\operatorname{Ext}\left(S, \mathrm{R}^{\prime}\right)$ is non-empty, $S$ is semi-algebraically connected if and only if $\operatorname{Ext}\left(S, \mathrm{R}^{\prime}\right)$ is semi-algebraically connected [9, Proposition 5.24].

\section{Proof of the Main Theorem}

Remark 3.1 Most of the techniques employed in the proof of the main theorem are similar to those found in [9], see [9, Sect. 13.1 and Sect. 13.3].

Throughout this section, $\mathrm{R}$ is a real closed field, $\mathcal{Q}, \mathcal{P}$ are finite subsets of $\mathrm{R}\left[X_{1}, \ldots, X_{k}\right]$, with $\operatorname{deg} P=d_{P}$ for all $P \in \mathcal{P}$, and $\operatorname{deg}(Q) \leq d_{0}$ for all $Q \in \mathcal{Q}$. We denote by $k^{\prime}$ the real dimension of $\operatorname{Zer}\left(\mathcal{Q}, \mathrm{R}^{k}\right)$. Let $Q=\sum_{F \in \mathcal{Q}} F^{2}$.

For $x \in \mathrm{R}^{k}$ and $r>0$, we will denote by $B_{k}(0, r)$ the open ball centered at $x$ of radius $r$. For any semi-algebraic subset $X \subset \mathrm{R}^{k}$, we denote by $\bar{X}$ the closure of $X$ in $\mathrm{R}^{k}$. It follows from the Tarski-Seidenberg transfer principle (see, for example, [9, Chap. 2, Sect. 5]) that the closure of a semi-algebraic set is again semi-algebraic.

We suppose using Proposition 2.17 that after making a linear change in coordinates if necessary the given system of coordinates is good with respect to $\operatorname{Zer}\left(Q, \mathrm{R}^{k}\right)$.

Using Proposition 2.22, suppose that $H \in \operatorname{Pos}_{\mathrm{R}, 2 d_{0}, k}$ satisfies

Property 3.2 For any $p, 0 \leq p \leq k, \mathrm{Cr}_{p}^{h}(H)$ defines a non-singular complete intersection in $\mathbb{P}_{\mathrm{C}}^{k}$.

Let $\operatorname{Def}(Q, H, \zeta)$ be defined by

$$
\operatorname{Def}(Q, H, \zeta)=(1-\zeta) Q-\zeta H .
$$

We first prove several properties of the polynomial $\operatorname{Def}(Q, H, \zeta)$. 
Proposition 3.3 Let $\tilde{\mathrm{R}}$ be any real closed field containing $\mathrm{R}\langle 1 / \Omega\rangle$, and let $C$ be a semi-algebraically connected component of

$$
\operatorname{Zer}\left(Q, \tilde{\mathrm{R}}^{k}\right) \cap B_{k}(0, \Omega)
$$

Then, there exists a semi-algebraically connected component, $D \subset \tilde{\mathrm{R}}\langle\zeta\rangle^{k}$, of the semi-algebraic set

$$
W=\left\{x \in B_{k}(0, \Omega) \mid \operatorname{Def}(Q, H, \zeta)(x) \leq 0\right\}
$$

such that $\bar{C}=\lim _{\zeta} D$.

Proof It is clear that $\operatorname{Zer}\left(Q, \tilde{\mathrm{R}}\langle\zeta\rangle^{k}\right) \cap B_{k}(0, \Omega) \subset W$, since $H(x) \geq 0$ for all $x \in$ $\tilde{\mathrm{R}}\langle\zeta\rangle^{k}$. Now let $C$ be a semi-algebraically connected component of $\operatorname{Zer}\left(Q, \tilde{\mathrm{R}}^{k}\right) \cap$ $B_{k}(0, \Omega)$ and let $D$ be the semi-algebraically connected component of $W$ containing $\operatorname{Ext}(C, \tilde{\mathrm{R}}\langle\zeta\rangle)$. Since $D$ is bounded over $\tilde{\mathrm{R}}$ and semi-algebraically connected, we have that $\lim _{\zeta} D$ is semi-algebraically connected (using, for example, Proposition 12.43 in [9]), and contained in $\operatorname{Zer}\left(Q, \tilde{\mathrm{R}}^{k}\right) \cap \overline{B_{k}(0, \Omega)}$. Moreover, $\lim _{\zeta} D$ contains $\bar{C}$. But $\bar{C}$ is a semi-algebraically connected component of $\operatorname{Zer}\left(Q, \tilde{\mathrm{R}}^{k}\right) \cap \overline{B_{k}(0, \Omega)}$ (using the conical structure at infinity of $\left.\operatorname{Zer}\left(Q, \tilde{\mathrm{R}}^{k}\right)\right)$, and hence $\lim _{\zeta} D=\bar{C}$.

Proposition 3.4 Let $\tilde{\mathrm{R}}$ be any real closed field containing $\mathrm{R}\langle 1 / \Omega\rangle$, and

$$
W=\operatorname{Zer}\left(\mathrm{Cr}_{k-k^{\prime}-1}(\operatorname{Def}(Q, H, \zeta)), \tilde{\mathrm{R}}\langle\zeta\rangle^{k}\right) \cap B_{k}(0, \Omega) .
$$

Then, $\lim _{\zeta} W=\operatorname{Zer}\left(Q, \tilde{\mathrm{R}}^{k}\right) \cap \overline{B_{k}(0, \Omega)}$.

We will use the following notation.

Notation 3.5 For $1 \leq p \leq q \leq k$, we denote by $\pi_{[p, q]}: \mathrm{R}^{k}=\mathrm{R}^{[1, k]} \rightarrow \mathrm{R}^{[p, q]}$ the projection

$$
\left(x_{1}, \ldots, x_{k}\right) \mapsto\left(x_{p}, \ldots, x_{q}\right) .
$$

Proof of Proposition 3.4 By Proposition 3.3, it is clear that $\lim _{\zeta} W \subset \operatorname{Zer}\left(Q, \tilde{\mathrm{R}}^{k}\right) \cap$ $\overline{B_{k}(0, \Omega)}$. We prove the other inclusion.

Let $V=\operatorname{Zer}\left(Q, \tilde{\mathrm{R}}^{k}\right)$, and suppose that $x \in \operatorname{reg} V^{(i)} \cap B_{k}(0, \Omega)$ for some $i, k-k^{\prime} \leq$ $i \leq k$. Every open semi-algebraic neighborhood $U$ of $x$ in $V \cap B_{k}(0, \Omega)$ contains a point $y \in \operatorname{reg} V^{\left(i^{\prime}\right)} \cap B_{k}(0, \Omega)$ for some $i^{\prime} \geq i$, such that the local dimension of $V$ at $y$ is equal to $d\left(i^{\prime}\right)$. Moreover, since the given system of coordinates is assumed to be good for $V$, we can also assume that the tangent space $T_{y}\left(\operatorname{reg} V^{\left(i^{\prime}\right)}\right)$ is transverse to the span of the first $k-d\left(i^{\prime}\right)$ coordinate vectors.

It suffices to prove that there exists $z \in W$ such that $\lim _{\zeta} z=y$. If this is true for every neighborhood $U$ of $x$ in $V$, this would imply that $x \in \lim _{\zeta} W$. 
Let $p=d\left(i^{\prime}\right)$. The property that $T_{y}\left(\operatorname{reg} V^{\left(i^{\prime}\right)}\right)$ is transverse to the span of the first $k-p$ coordinate vectors implies that $y$ is an isolated point of $V \cap \pi_{[k-p+1, k]}^{-1}(y)$. Let $T \subset \tilde{\mathrm{R}}\langle\zeta\rangle^{k}$ denote the semi-algebraic subset of $B_{k}(0, \Omega)$ defined by

$$
T=\left\{x \in B_{k}(0, \Omega) \mid \operatorname{Def}(Q, H, \zeta)(x) \leq 0\right\},
$$

and $D_{y}$ denote the semi-algebraically connected component of $T \cap \pi_{[k-p+1, k]}^{-1}(y)$ containing $y$. Then, $D_{y}$ is a closed and bounded semi-algebraic set, with $\lim _{\zeta} D_{y}=y$. The boundary of $D_{y}$ is contained in

$$
\operatorname{Zer}\left(\operatorname{Def}(Q, H, \zeta), \tilde{\mathrm{R}}\langle\zeta\rangle^{k}\right) \cap \pi_{[k-p+1, k]}^{-1}(y) .
$$

Let $z \in D_{y}$ be a point in $D_{y}$ for which the $(k-p)$ th coordinate achieves its maximum. Then, $z \in \operatorname{Zer}\left(\operatorname{Cr}_{k-p-1}(\operatorname{Def}(Q, H, \zeta)), \tilde{\mathrm{R}}\langle\zeta\rangle^{k}\right)$, and since $p \leq k^{\prime}$,

$$
\operatorname{Zer}\left(\operatorname{Cr}_{k-p-1}(\operatorname{Def}(Q, H, \zeta)), \tilde{\mathrm{R}}\langle\zeta\rangle^{k}\right) \subset \operatorname{Zer}\left(\operatorname{Cr}_{k-k^{\prime}-1}(\operatorname{Def}(Q, H, \zeta)), \tilde{\mathrm{R}}\langle\zeta\rangle^{k}\right)
$$

and hence, $z \in W$. Moreover, $\lim _{\zeta} z=y$.

Proposition 3.6 Let $\tilde{\mathrm{R}}$ be any real closed field containing $\mathrm{R}$ and $\tilde{\mathrm{C}}$ the algebraic closure of $\tilde{\mathrm{R}}$. For every $p, 0 \leq p \leq k, \mathrm{Cr}_{p}^{h}(\operatorname{Def}(Q, H, \zeta))$ defines a non-singular complete intersection in $\mathbb{P}_{\tilde{\mathrm{C}}\langle\zeta\rangle}^{k}$.

Proof By Property 3.2 of $H$, we have that for each $p, 0 \leq p \leq k, \mathrm{Cr}_{p}^{h}(H)$ defines a non-singular complete intersection in $\mathbb{P}_{\tilde{\mathrm{C}}}^{k}$. Thus, for each $p, 0 \leq p \leq k$, $\operatorname{Cr}_{p}^{h}(\operatorname{Def}(Q, H, 1))$ defines a non-singular complete intersection in $\mathbb{P}_{\tilde{\mathrm{C}}}^{k}$. Since the property of being non-singular complete intersection is first-order expressible, the set of $t \in \tilde{\mathrm{C}}$ for which this holds is constructible, and since the property is also stable there is an open subset containing 1 for which it holds. But since a constructible subset of $\tilde{C}$ is either finite or co-finite, there exists an open interval to the right of 0 in $\tilde{R}$ for which the property holds, and in particular it holds for infinitesimal $\zeta$.

Proposition 3.7 Let $\sigma \in\{0,+1,-1\}^{\mathcal{P}}$, and let $C$ be a semi-algebraically connected component of $\mathcal{R}\left(\sigma, \operatorname{Zer}\left(Q, \mathrm{R}\langle 1 / \Omega\rangle^{k}\right) \cap B_{k}(0, \Omega)\right)$. Then, there exists a unique semialgebraically connected component, $D \subset \mathrm{R}\langle 1 / \Omega, \varepsilon, \delta\rangle^{k}$, of the semi-algebraic set defined by

$$
(Q=0) \wedge \bigwedge_{\substack{P \in \mathcal{P} \\ \sigma(P)=0}}(-\delta<P<\delta) \wedge \bigwedge_{\substack{P \in \mathcal{P} \\ \sigma(P)=1}}(P>\varepsilon) \wedge \bigwedge_{\substack{P \in \mathcal{P} \\ \sigma(P)=-1}}(P<-\varepsilon) \wedge\left(|X|^{2}<\Omega^{2}\right)
$$

such that $C=D \cap \mathrm{R}\langle 1 / \Omega\rangle^{k}$. Moreover, if $C$ is a semi-algebraically connected component of $\mathcal{R}\left(\sigma, \operatorname{Zer}\left(Q, \mathrm{R}\langle 1 / \Omega\rangle^{k}\right) \cap B_{k}(0, \Omega)\right), C^{\prime}$ is a semi-algebraically connected component of $\mathcal{R}\left(\sigma^{\prime}, \operatorname{Zer}\left(Q, \mathrm{R}\langle 1 / \Omega\rangle^{k}\right) \cap B_{k}(0, \Omega)\right)$, and $D, D^{\prime}$ are the unique semialgebraically connected components as above satisfying $C=D \cap \mathrm{R}\langle 1 / \Omega\rangle^{k}, C^{\prime}=$ $D^{\prime} \cap \mathrm{R}\langle 1 / \Omega\rangle^{k}$, then we have $\bar{D} \cap \overline{D^{\prime}}=\emptyset$ if $C \neq C^{\prime}$. 
Proof The first part is clear. To prove the second part, suppose, seeking a contradiction, that $x \in \bar{D} \cap \overline{D^{\prime}}$. Notice that $\lim _{\delta} x \in \operatorname{Ext}(C, \mathrm{R}\langle 1 / \Omega, \varepsilon\rangle) \cap \operatorname{Ext}\left(C^{\prime}, \mathrm{R}\langle 1 / \Omega, \varepsilon)\right.$, but $\operatorname{Ext}(C, \mathrm{R}\langle 1 / \Omega, \varepsilon\rangle) \cap \operatorname{Ext}\left(C^{\prime}, \mathrm{R}\langle 1 / \Omega, \varepsilon\rangle\right)=\emptyset$ since $C \cap C^{\prime}=\emptyset$.

We denote by $\mathrm{R}^{\prime}$ the real closed field $\mathrm{R}\langle 1 / \Omega, \varepsilon, \delta\rangle$ and by $\mathrm{C}^{\prime}$ the algebraic closure of $\mathrm{R}^{\prime}$.

We also denote

$$
G=\sum_{i=1}^{k} X_{i}^{2}-\Omega^{2}
$$

Let $\mathcal{P}^{\prime} \subset \mathrm{R}^{\prime}\left[X_{1}, \ldots, X_{k}\right]$ be defined by

$$
\mathcal{P}^{\prime}=\bigcup_{P \in \mathcal{P}}\{P \pm \varepsilon, P \pm \delta\} \cup\{G\} .
$$

By Proposition 3.7, we will henceforth restrict attention to strict sign conditions on the family $\mathcal{P}^{\prime}$.

Let $\mathcal{H}=\left(H_{F} \in \operatorname{Pos}_{\mathrm{R}^{\prime}, \operatorname{deg}(F), k}\right)_{F \in \mathcal{P}^{\prime}}$ be a family of polynomials with generic coefficients. More precisely, this means that $\mathcal{H}$ is chosen so that it avoids a certain Zariski-closed subset of the product $\times_{F \in \mathcal{P}^{\prime}} \operatorname{Pos}_{\mathrm{R}^{\prime}\langle\zeta\rangle, \operatorname{deg}(F), k}$ of codimension at least one, defined by the condition that

$$
\mathrm{Cr}_{k-k^{\prime}-1}^{h}(\operatorname{Def}(Q, H, \zeta)) \cup \bigcup_{F \in \mathcal{P}^{\prime \prime}}\left\{H_{F}^{h}\right\}
$$

is not a non-singular, complete intersection in $\mathbb{P}_{\mathrm{C}^{\prime}\langle\zeta\rangle}^{k}$ for some $\mathcal{P}^{\prime \prime} \subset \mathcal{P}^{\prime}$.

Proposition 3.8 For each $j, 0 \leq j \leq k^{\prime}$, and subset $\mathcal{P}^{\prime \prime} \subset \mathcal{P}^{\prime}$ with card $\mathcal{P}^{\prime \prime}=j$, and $\tau \in\{-1,+1\}^{\mathcal{P}^{\prime \prime}}$ the set of homogeneous polynomials

$$
\operatorname{Cr}_{k-k^{\prime}-1}^{h}(\operatorname{Def}(Q, H, \zeta)) \cup \bigcup_{F \in \mathcal{P}^{\prime \prime}}\left\{\left(1-\varepsilon^{\prime}\right) F^{h}-\tau(F) \varepsilon^{\prime} H_{F}^{h}\right\}
$$

defines a non-singular, complete intersection in $\mathbb{P}_{C^{\prime}\left\langle\zeta, \varepsilon^{\prime}\right\rangle}^{k}$.

Proof Consider the family of polynomials,

$$
\mathrm{Cr}_{k-k^{\prime}-1}^{h}(\operatorname{Def}(Q, H, \zeta)) \cup \bigcup_{F \in \mathcal{P}^{\prime \prime}}\left\{(1-t) F^{h}-\tau(F) t H_{F}^{h}\right\}
$$

obtained by substituting $t$ for $\varepsilon^{\prime}$ in the given system. Since, by Proposition 3.6, the set $\mathrm{Cr}_{k-k^{\prime}-1}^{h}(\operatorname{Def}(Q, H, \zeta))$ defines a non-singular complete intersection in $\mathbb{P}_{\mathrm{C}^{\prime}\langle\zeta\rangle}$, and the $H_{F}$ 's are chosen generically, the above system defines a non-singular complete intersection in $\mathbb{P}_{\mathrm{C}^{\prime}\langle\zeta\rangle}$ when $t=1$. The set of $t \in \mathrm{C}^{\prime}\langle\zeta\rangle$ for which the above system defines a non-singular, complete intersection is constructible, contains 1 , and since being a non-singular, complete intersection is a stable condition, it is co-finite. Hence, it must contain an open interval to the right of 0 in $\mathrm{R}^{\prime}\langle\zeta\rangle$, and hence in particular if we 
substitute the infinitesimal $\varepsilon^{\prime}$ for $t$ we obtain that the system defines a non-singular, complete intersection in $\mathbb{P}_{\mathrm{C}^{\prime}\left\langle\zeta, \varepsilon^{\prime}\right\rangle}^{k}$.

Proposition 3.9 Let $\tau \in\{+1,-1\}^{\mathcal{P}^{\prime}}$ with $\tau(F)=-1$, if $F=G(3.1)$, and let $C$ be a semi-algebraically connected component of $\mathcal{R}\left(\tau, \operatorname{Zer}\left(Q, \mathrm{R}^{\prime k}\right)\right)$.

Then, there exist a subset $\mathcal{P}^{\prime \prime} \subset \mathcal{P}^{\prime}$ with card $\mathcal{P}^{\prime \prime} \leq k^{\prime}$ and a bounded semialgebraically connected component $D$ of the algebraic set

$$
\operatorname{Zer}\left(\operatorname{Cr}_{k-k^{\prime}-1}(\operatorname{Def}(Q, H, \zeta)) \cup \bigcup_{F \in \mathcal{P}^{\prime \prime}}\left\{\left(1-\varepsilon^{\prime}\right) F-\tau(F) \varepsilon^{\prime} H_{F}\right\}, \mathrm{R}^{\prime}\left\langle\zeta, \varepsilon^{\prime}\right\rangle^{k}\right)
$$

such that $\lim _{\zeta} D \subset \bar{C}$, and $\lim _{\zeta} D \cap C \neq \emptyset$.

Proof Let $V=\operatorname{Zer}\left(Q, \mathrm{R}^{\prime k}\right)$. By Proposition 3.4, with $\tilde{\mathrm{R}}$ substituted by $\mathrm{R}^{\prime}$, we have that, for each $x \in C$, there exists $y \in \operatorname{Zer}\left(\mathrm{Cr}_{k-k^{\prime}-1}\left(\operatorname{Def}(Q, H, \zeta), \mathrm{R}^{\prime}\langle\zeta\rangle^{k}\right)\right.$ such that $\lim _{\zeta} y=x$.

Moreover, using the fact that $\mathcal{R}\left(\tau, \mathrm{R}^{\prime k}\right)$ is open we have that

$$
y \in \operatorname{Zer}\left(\mathrm{Cr}_{k-k^{\prime}-1}(\operatorname{Def}(Q, H, \zeta)), \mathrm{R}^{\prime}\langle\zeta\rangle^{k}\right) \cap \mathcal{R}\left(\tau, \mathrm{R}^{\prime k}\right) .
$$

Thus, there exists a semi-algebraically connected component $C^{\prime}$ of

$$
\operatorname{Zer}\left(\mathrm{Cr}_{k-k^{\prime}-1}(\operatorname{Def}(Q, H, \zeta)), \mathrm{R}^{\prime}\langle\zeta\rangle^{k}\right) \cap \mathcal{R}\left(\tau, \mathrm{R}^{\prime k}\right)
$$

such that $\lim _{\zeta} C^{\prime} \subset \bar{C}$, and $\lim _{\zeta} C^{\prime} \cap C \neq \emptyset$.

Note that the closure $\bar{C}$ is a semi-algebraically connected component of

$$
\mathcal{R}\left(\bar{\tau}, \operatorname{Zer}\left(Q, \mathrm{R}^{\prime k}\right)\right),
$$

where $\bar{\tau}$ is the formula $\bigwedge_{F \in \mathcal{P}^{\prime}}(\tau(F) F \geq 0)$.

The proof of the proposition now follows the proof of Proposition 13.2 in [9], and uses the fact that the set of polynomials $\bigcup_{F \in \mathcal{P}^{\prime}}\left\{\left(1-\varepsilon^{\prime}\right) F-\tau(F) \varepsilon^{\prime} H_{P}\right\}$ has the property that no $k^{\prime}+1$ of distinct elements of this set can have a common zero in $\operatorname{Zer}\left(\mathrm{Cr}_{k-k^{\prime}-1}(\operatorname{Def}(Q, H, \zeta)), \mathrm{R}^{\prime}\left\langle\zeta, \varepsilon^{\prime}\right\rangle^{k}\right)$ by Proposition 3.8 .

Proof of Theorem 1.1 Using the conical structure at infinity of semi-algebraic sets, we have the following equality:

$$
\begin{aligned}
& \sum_{\sigma \in\{-1,1,0\} \mathcal{P}} b_{0}\left(\mathcal{R}\left(\sigma, \operatorname{Zer}\left(\mathcal{Q}, \mathrm{R}^{k}\right)\right)\right) \\
= & \sum_{\sigma \in\{-1,1,0\}} b^{\mathcal{P}} b_{0}\left(\mathcal{R}\left(\sigma, \operatorname{Zer}\left(\mathcal{Q}, \mathrm{R}\langle 1 / \Omega\rangle^{k}\right) \cap B_{k}(0, \Omega)\right)\right) .
\end{aligned}
$$

By Proposition 3.7, it suffices to bound the number of semi-algebraically connected components of the realizations $\mathcal{R}\left(\tau, \operatorname{Zer}\left(Q, \mathrm{R}^{\prime k}\right) \cap B_{k}(0, \Omega)\right)$, where $\tau \in$ $\{-1,1\}^{\mathcal{P}^{\prime}}$ satisfying

$$
\tau(F)=-1 \quad \text { if } F=G,
$$




$$
\begin{aligned}
& \tau(F)=1 \quad \text { if } F=P-\varepsilon \text { or } F=P+\delta \text { for some } P \in \mathcal{P}, \\
& \tau(F)=-1 \quad \text { otherwise. }
\end{aligned}
$$

Using Proposition 3.9, it suffices to bound the number of semi-algebraically connected components which are bounded over $\mathrm{R}^{\prime}$ of the real algebraic sets

$$
\operatorname{Zer}\left(\mathrm{Cr}_{k-k^{\prime}-1}(\operatorname{Def}(Q, H, \zeta)) \cup \bigcup_{F \in \mathcal{P}^{\prime \prime}}\left\{\left(1-\varepsilon^{\prime}\right) F+\tau(F) \varepsilon^{\prime} H_{F}\right\}, \mathrm{R}^{\prime}\left\langle\zeta, \varepsilon^{\prime}\right\rangle^{k}\right)
$$

for all $\mathcal{P}^{\prime \prime} \subset \mathcal{P}^{\prime}$ with card $\mathcal{P}^{\prime \prime}=j \leq k^{\prime}$ and all $\tau \in\{-1,1\}^{\mathcal{P}^{\prime \prime}}$ satisfying (3.2).

Using Theorem 2.6, we get that the number of such components is bounded by

$$
\left(\begin{array}{c}
k+1 \\
k-k^{\prime}+\# \mathcal{P}^{\prime \prime}+1
\end{array}\right)\left(2 d_{0}\right)^{k-k^{\prime}} d_{\mathcal{P}^{\prime \prime}} \max _{F \in \mathcal{P}^{\prime \prime}}\left\{2 d_{0}, \operatorname{deg}(F)\right\}^{k^{\prime}-\# \mathcal{P}^{\prime \prime}}+2\left(k-\# \mathcal{P}^{\prime \prime}+1\right),
$$

where $d_{\mathcal{P}^{\prime \prime}}=\prod_{F \in \mathcal{P}^{\prime \prime}} \operatorname{deg}(F)$.

Each $F \in \mathcal{P}^{\prime} \backslash\{G\}$ is of the form $F \in\{P \pm \varepsilon, P \pm \delta\}$ for some $P \in \mathcal{P}$, and the algebraic sets defined by each of these four polynomials are disjoint. Thus, we have that

$$
\sum_{\sigma \in\{0,1,-1\}^{\mathcal{P}}} b_{0}\left(\mathcal{R}\left(\sigma, \operatorname{Zer}\left(Q, \mathrm{R}^{k}\right)\right)\right)
$$

is bounded by

$$
\sum_{\substack{\mathcal{I} \subset \mathcal{P} \\
\# \mathcal{I} \leq k^{\prime}}} 4^{\# \mathcal{I}}\left(\left(\begin{array}{c}
k+1 \\
k-k^{\prime}+\# \mathcal{I}+1
\end{array}\right)\left(2 d_{0}\right)^{k-k^{\prime}} d_{\mathcal{I}} \max _{P \in \mathcal{I}}\left\{2 d_{0}, d_{P}\right\}^{k^{\prime}-\# \mathcal{I}}+2(k-\# \mathcal{I}+1)\right) .
$$

\section{Applications}

There are several applications of the bound on the number of semi-algebraically connected components of sign conditions of a family of real polynomials in discrete geometry. We discuss below an application for bounding the number of geometric permutations of $n$ well separated convex bodies in $\mathbb{R}^{d}$ induced by $k$-transversals.

In [15], the authors reduce the problem of bounding the number of geometric permutations of $n$ well separated convex bodies in $\mathbb{R}^{d}$ induced by $k$-transversals to bounding the number of semi-algebraically connected components realizable sign conditions of

$$
\left(\begin{array}{c}
2^{k+1}-2 \\
k
\end{array}\right)\left(\begin{array}{c}
n \\
k+1
\end{array}\right)
$$

polynomials in $d^{2}$ variables, where each polynomial has degree at most $2 k$, on an algebraic variety (the real Grassmannian of $k$-planes in $\mathbb{R}^{d}$ ) in $\mathbb{R}^{d^{2}}$ defined by polynomials of degree 2 . The real Grassmannian has dimension $k(d-k)$. Applying Theorem 1.1, we obtain that the number of semi-algebraically connected components of 
all realizable sign conditions in this case is bounded by

$$
\left(k\left(\begin{array}{c}
2^{k+1}-2 \\
k
\end{array}\right)\left(\begin{array}{c}
n \\
k+1
\end{array}\right)\right)^{k(d-k)}(O(1))^{d^{2}},
$$

which is a strict improvement of the bound,

$$
\left(\left(\begin{array}{c}
2^{k+1}-2 \\
k
\end{array}\right)\left(\begin{array}{c}
n \\
k+1
\end{array}\right)\right)^{k(d-k)}(O(k))^{d^{2}},
$$

in [15, Theorem 2] (especially in the case when $k$ is close to $d$ ).

As mentioned in the introduction, our bound might also have some relevance in a new method which has been developed for bounding the number of incidences between points and algebraic varieties of constant degree, using a decomposition technique based on the polynomial ham-sandwich cut theorem [16, 17, 22, 27].

Acknowledgements The authors would like to thank J. Matousek for bringing this problem to their attention and for providing encouragement and useful advice, and M.-F. Roy for making helpful comments on a first draft of the paper. The authors were partially supported by an NSF grant CCF-0915954.

\section{References}

1. Alon, N.: Tools from higher algebra. In: Handbook of Combinatorics, vol. 2, pp. 1749-1783. Elsevier, Amsterdam (1995)

2. Bank, B., Giusti, M., Heintz, J., Mbakop, G.M.: Polar varieties, real equation solving, and data structures: the hypersurface case. J. Complex. 13(1), 5-27 (1997)

3. Bank, B., Giusti, M., Heintz, J., Safey El Din, M., Schost, E.: On the geometry of polar varieties. Appl. Algebra Eng. Commun. Comput. 21, 33-83 (2010). doi:10.1007/s00200-009-0117-1

4. Basu, S., Kettner, M.: A sharper estimate on the Betti numbers of sets defined by quadratic inequalities. Discrete Comput. Geom. 39(4), 734-746 (2008)

5. Basu, S., Pollack, R., Roy, M.-F.: On the Betti numbers of sign conditions. Proc. Am. Math. Soc. 133(4), 965-974 (2005) (electronic)

6. Basu, S., Pollack, R., Roy, M.-F.: On the combinatorial and algebraic complexity of quantifier elimination. J. ACM 43(6), 1002-1045 (1996)

7. Basu, S., Pollack, R., Roy, M.-F.: On computing a set of points meeting every cell defined by a family of polynomials on a variety. J. Complex. 13(1), 28-37 (1997)

8. Basu, S., Pollack, R., Roy, M.-F.: Betti number bounds, applications and algorithms. In: Current Trends in Combinatorial and Computational Geometry: Papers from the Special Program at MSRI. MSRI Publications, vol. 52, pp. 87-97. Cambridge University Press, Cambridge (2005)

9. Basu, S., Pollack, R., Roy, M.-F.: Algorithms in Real Algebraic Geometry, 2nd edn. Algorithms and Computation in Mathematics, vol. 10. Springer, Berlin (2006)

10. Basu, S., Pollack, R., Roy, M.-F.: An asymptotically tight bound on the number of connected components of realizable sign conditions. Combinatorica 29, 523-546 (2009)

11. Benedetti, R., Loeser, F., Risler, J.-J.: Bounding the number of connected components of a real algebraic set. Discrete Comput. Geom. 6(3), 191-209 (1991)

12. Bochnak, J., Coste, M., Roy, M.-F.: Géométrie Algébrique Réelle. Ergebnisse der Mathematik und ihrer Grenzgebiete, vol. 12. Springer, Berlin (1987). (Second edition in English: Real Algebraic Geometry. Results in Mathematics and Related Areas, vol. 36. Springer, Berlin (1998))

13. Chistov, A., Fournier, H., Gurvits, L., Koiran, P.: Vandermonde matrices, NP-completeness and transversal subspaces. Found. Comput. Math. 3(4), 421-427 (2003)

14. Safey El Din, M., Trebuchet, P.: Strong bi-homogeneous Bézout theorem and its use in effective real algebraic geometry. http://arxiv.org/abs/cs/0610051 (2006) 
15. Goodman, J.E., Pollack, R., Wenger, R.: Bounding the number of geometric permutations induced by $k$-transversals. J. Comb. Theory, Ser. A 75(2), 187-197 (1996)

16. Guth, L., Katz, N.H.: On the Erdös distinct distance problem in the plane. arXiv: $1011.4105 \mathrm{v} 1$ [math.CO] (2011)

17. Kaplan, H., Matousek, J., Safernova, Z., Sharir, M.: Unit distances in three dimensions. arXiv: 1107.1077v1 [math.CO] (2011)

18. Matousek, J.: Private correspondence (2011)

19. Milnor, J.: On the Betti numbers of real varieties. Proc. Am. Math. Soc. 15, 275-280 (1964)

20. Petrovskiı̆, I.G., Oleĭnik, O.A.: On the topology of real algebraic surfaces. Izv. Akad. Nauk SSSR, Ser. Mat. 13, 389-402 (1949)

21. Pollack, R., Roy, M.-F.: On the number of cells defined by a set of polynomials. C. R. Math. 316, 573-577 (1993)

22. Solymosi, J., Tao, T.: An incidence theorem in higher dimensions. arXiv:1103.2926v2 [math.CO]

23. Thom, R.: Sur l'homologie des variétés algébriques réelles. In: Differential and Combinatorial Topology (A Symposium in Honor of Marston Morse), pp. 255-265. Princeton University Press, Princeton (1965)

24. Viro, O.Y., Fuchs, D.B.: Homology and cohomology. In: Topology. II. Encyclopaedia Math. Sci., vol. 24, pp. 95-196. Springer, Berlin (2004). Translated from the Russian by C.J. Shaddock

25. Voisin, C.: Hodge Theory and Complex Algebraic Geometry. II. Cambridge Studies in Advanced Mathematics, vol. 77. Cambridge University Press, Cambridge (2007). English edition, Translated from the French by Leila Schneps

26. Warren, H.E.: Lower bounds for approximation by nonlinear manifolds. Trans. Am. Math. Soc. 133, 167-178 (1968)

27. Zahl, J.: An improved bound on the number of point-surface incidences in three dimensions. arXiv: 1104.4987v3 [math.CO] (2011) 